\title{
GESTIÓN DE TALENTO HUMANO Y SATISFACCIÓN LABORAL EN BANCOS DE GUAYAQUIL
}

\author{
HUMAN TALENT MANAGEMENT AND JOB SATISFACTION IN BANKS OF \\ GUAYAQUIL
}

\author{
Priscilla Rossana PAREDES FLORIL ${ }^{1}$ \\ Juan José BUSTAMANTE VILLEGAS ${ }^{2}$
}

\begin{tabular}{|lll|}
\hline Recibido & $:$ & 31.03 .2021 \\
Aceptado & $:$ & 15.06 .2021 \\
Publicado & $:$ & 02.08 .2021 \\
\hline
\end{tabular}

\begin{abstract}
RESUMEN: Este estudio consideró como objetivo establecer la conexión que existe entre gestión de talento humano y la satisfacción laboral en los bancos con mayor capital financiero de Guayaquil. La investigación fue cuantitativa, método inferencial, diseño descriptivo-correlacional. Se utilizó cuestionario con escala de Likert de 40 ítems para recaudar información, la muestra poblacional fue 370 empleados. Obteniendo en el análisis de fiabilidad un valor significativo de 0.911. En la prueba de KMO y Bartlett dio un resultado significativo $<0.05$, se utilizó para la extracción de datos, el método de rotación, Varimax de Kaiser, quedando convergido 10 iteraciones en la matriz de varianza factorial exploratoria. La prueba de Chi-Cuadrado permitió comprobar la probabilidad de las correlaciones entre las hipótesis con una estimación relevante $\mathrm{p}<0.05$. En el Análisis Factorial Confirmatorio se utilizó la gráfica de path diagram del programa SPSS AMOS 26.0 para establecer las estimaciones factoriales de variables latentes cuyos valores absolutos fueron $>0.3$, concluyendo que se logró comprobar la conexión positiva entre gestión de talento humano y satisfacción laboral de los empleados en los bancos con mayor capital financiero de Guayaquil.
\end{abstract}

Palabras clave: Integración, gestión, organización, satisfacción, formación.

ABSTRACT: This study considered establishing the connection between human talent management and job satisfaction in the banks with the largest financial capital of Guayaquil. The research was quantitative, under the inferential method, and of a descriptive-correlational design. A 40-item Likert questionnaire was used to collect information. The population sample was 370 employees. A significant value of 0.911 was obtained in the reliability analysis. In the $\mathrm{KMO}$ and Bartlett test it gave a significant result $<0.05$. The Kaiser's Varimax rotation method was executed for data extraction, with 10 iterations converging in the exploratory factor variance matrix. The Chi-Square test allowed to check the probability of the correlations between the hypotheses with a relevant estimate $p<0.05$. In the Confirmatory Factor Analysis, the path diagram graph of the SPSS AMOS 26.0 program was used to establish the factorial estimates of latent variables whose absolute values were $>0.3$, concluding that it was possible to verify the positive connection between management of human talent and job satisfaction of employees in the banks with the largest financial capital of Guayaquil.

Keywords: Integration, Management, Organization, Satisfaction, Training.

\footnotetext{
${ }^{1}$ Doctora en Ciencias Administrativas. Universidad Politécnica Salesiana - Carrera Contabilidad y Auditoría. Guayaquil, Ecuador. Email: pparedes@ ups.edu.ec. ORCID: https://orcid.org/0000-0001-9870-1339

${ }_{2}^{2}$ Magíster en Administración de Empresas. Universidad Politécnica Salesiana. Guayaquil, Ecuador. Email: juan_100501@hotmail.com. ORCID: https://orcid.org/0000-0002-2729-0074
} 


\section{Journal of the Academy $|45|$}

\section{INTRODUCCIÓN}

Esta investigación nace del deseo de proponer indicadores correlacionales que existen entre la gestión de talento humano y satisfacción laboral en los bancos con mayor capital financiero de Guayaquil, con base a la realidad, se busca cumplir con las demandas de las exigencias actuales, contribuyendo de manera eficaz en la cultura organizacional. Por otro lado, la sensación de bienestar de los colaboradores es el inicio del rendimiento y productividad de la institución, para así comprender en que consiste la satisfacción laboral y la importancia que implica para el manejo de una organización (Grijalva et al., 2017).

Al investigar la liquidez específicamente de los bancos privados, se evidencia un acrecentamiento en el grupo de bancos pequeños y medianos, luego que en los bancos grandes se mantuvo sin inquietud, sin embargo, hasta noviembre del 2020, los bancos con mayor capital financiero de Guayaquil según la Superintendencia de Bancos del Ecuador (2020) son los siguientes (Ver tabla1).

\section{Tabla 1}

Ranking de los diez bancos con más utilidades

\begin{tabular}{lll}
\hline Posición & Institución & Utilidades \\
\hline 1 & Banco Pichincha & $\$ 44.5$ millones \\
2 & Banco Internacional & $\$ 28.2$ millones \\
3 & Banco Guayaquil & $\$ 25.8$ millones \\
4 & Banco Bolivariano & $\$ 25.2$ millones \\
5 & Banco Pacífico & $\$ 24.8$ millones \\
6 & Banco Diners & $\$ 20$ millones \\
7 & Banco Produbanco & $\$ 15$ millones \\
8 & Banco del Austro & $\$ 8.6$ millones \\
9 & Banco General Rumiñahui & $\$ 6.6$ millones \\
10 & Banco Citibank & $\$ 5$ millones \\
\hline
\end{tabular}

Fuente: Elaboración Propia en base a El Universo (2020).

Surge la interrogante: ¿De qué manera la gestión de talento humano se relaciona con la satisfacción laboral de los empleados de los bancos con mayor capital financiero de Guayaquil? 


\section{Journal of the Academy $|46|$}

\section{DESARROLLO}

\section{Gestión de Talento Humano}

La gestión de talento humano (GTH) es una agrupación integrada de procedimientos, estrategias, técnicas, métodos, organización, administración de las instituciones, creados para gestionar de manera satisfactoria un constructo de procesos que permiten lograr la productividad empresarial, (Pérez, 2016). Por otro lado, Montoya y Boyero (2016) en sentido político indican que son funciones fundamentales de la organización, para desarrollar eficazmente una dirección con bases políticas, técnicas.

Por consiguiente, en el presente estudio se consideraron las siguientes dimensiones para la variable 1: Dimensión1: Integración: Para, Montoya y Boyero (2016) son procedimientos que permiten a las organizaciones resolver sus necesidades de medios humanos, como sus beneficios, estabilidad, definir sus necesidades y capacitación. Dimensión 2: Organización: Según, Garbanzo (2015) es una actividad realizada bajo coordinación con lineamientos específicos para alcanzar una meta propuesta. Dimensión 3: Formación: Martínez (2011) la considera como procesos que intervienen eficazmente en el bienestar del personal con base de contenidos actitudinal, procedimental y conceptual. Dimensión 4: Retención: Prieto (2013) manifiesta que es importante inferir para integrar al personal y lograr metas significativas para la organización.

Del mismo modo, existen varios modelos de GTH (Ver tabla 2), que permiten a los interesados interactuar de manera interna y externa en la organización, así como también satisfacer sus necesidades acordes a los intereses comunes. A continuación, se demuestra mediante una tabla los diferentes modelos de GTH propuesto por (Mondragón et al., 2017; Cruz et al., 2002).

\section{Tabla 2}

Modelos de Gestión de Talento Humano

\begin{tabular}{lll}
\hline Modelos / Autor- Año & DESCRIPCIÓN \\
\hline $\begin{array}{l}\text { Modelo de Werther } \\
\text { Davis (1996) }\end{array}$ & $\begin{array}{l}\text { Concibe a la organización como un sistema compuesto } \\
\text { por varias áreas que mantienen una sinergia e interactúan } \\
\text { entre sí. }\end{array}$
\end{tabular}


Journal of the Academy $\mid 47$ |

Modelo de Zayas (1996)

Interdependencia entre: organización, selección y desarrollo de personal.

Los procesos: admisión, reclutamiento y selección, retención, desarrollo laboral

Modelo de GTH de Cuatro subsistemas: Flujo de talento humano, Formación

Diagnóstico, Proyección y y desarrollo, Organismos laborales, y Compensación.

Control

Modelo de Beer

Asocia los factores funcionales, estructurales, tecnológicos, dinámicos que caracterizan metodología empleadas al conocimiento

Modelo de Gestión en Administración de talento humano es un órgano de Línea o Staff, "Staff".

Modelo de Gestión por Identificación de competencias organizacionales.

Competencia,

Modelo de Gestión basado

Capital humano como el único eje que interviene en las capacidades individuales del ser humano, siendo el en el Talento mismo promotor de la inversión.

Fuente: Elaboración Propia (2020)

En ese entorno se ejecuta la Escuela de las Ciencias Administrativas, la cual abarca distintos enfoques y teorías, cabe destacar las más relevantes para este estudio: el enfoque de sistema, Teoría Z, la teoría de contingencias, teoría de relaciones humanas, y la teoría del control de calidad, de los recursos humanos, (Manjarrez et al., 2016).

Diversos autores como Daza (2017), Ramos (2018) y Ávila (2018) en sus estudios cuantitativos y correlacionales determinaron una conexión directa entre las variables GTH y satisfacción laboral. Es importante señalar que cuando el estado de ánimo del personal es satisfactorio se garantiza una estabilidad laboral (Chacón, 2016).

\section{Satisfacción Laboral}

La satisfacción es un predictor muy importante de conductas disfuncionales dentro de la organización, ésta es entendida como un estado emocional que proyecta una relación afectiva en el trabajo así lo manifiesta (Chiang y Ojeda, 2013; Pilatasig, 2020). Para Cruz (2018) es el bienestar y seguridad que se le brinda a los empleados para lograr un mejor rendimiento laboral dentro del entorno de la organización. 
En este contexto, se determinaron las siguientes dimensiones: Dimensión 1: Aspecto cognoscitivo son orientaciones psicológicas que estudia el comportamiento de la mente humana considerando al empleado como ente productivo capaz de construir, modificar, diversificar y coordinar esquemas de conocimiento enriqueciendo el conocimiento personal (Mejía y Tómala, 2017). Dimensión 2: Aspecto afectivo, es la capacidad que tiene el individuo en reaccionar ante los estímulos extrínsecos e intrínsecos, entre las expresiones importantes tenemos los sentimientos y las emociones, que provocan sensaciones como: amor-desamor, alegría-tristeza, aceptación-rechazo, así lo manifiestan (Meneses y Bendezú, 2017). Dimensión 3: Aspecto conductual se apropia de las experiencias que vive, se interesa en lo aprende. (Barros et al., 2017)

Con la finalidad de cimentar la importancia de la variable basado en los criterios de (Daza, 2017; Manene, 2012; Escobar, 2019) se consideran las teorías que fundamentan la satisfacción laboral de manera directa para impulsar motivación y productividad dentro de la empresa (Ver tabla 3).

\section{Tabla 3}

Teorías de Satisfacción Laboral

\begin{tabular}{|c|c|c|}
\hline AUTOR-AÑO & TEORÍAS & CARACTERÍSTICAS \\
\hline $\begin{array}{l}\text { Herzberg, } \\
\text { Mausner } \\
\text { Snyderman, } \\
\text { (1959) }\end{array}$ & $\begin{array}{l}\text { Teoría } \\
\text { bifactorial }\end{array}$ & $\begin{array}{l}\text { Pretende analizar el comportamiento de los seres humano según las } \\
\text { necesidades de su desarrollo e interrelación a través de las teorías que } \\
\text { provocan satisfacción e insatisfacción, (Manene, 2012). }\end{array}$ \\
\hline Adams, (1963) & $\begin{array}{l}\text { Teoría de } \\
\text { la equidad }\end{array}$ & $\begin{array}{l}\text { Comprende la satisfacción e insatisfacción como equilibrio y desequilibrio } \\
\text { en las personas que relacionan dentro de la institución empresarial, (Daza, } \\
2017 \text { ). }\end{array}$ \\
\hline $\begin{array}{l}\text { Quarstein, } \\
\text { Mcafee }\end{array}$ & $\begin{array}{l}\text { Teoría de } \\
\text { eventos o }\end{array}$ & $\begin{array}{l}\text { Proviene de las actitudes emocionales, y sentimentales tanto de factores } \\
\text { intrínsecos, como de factores extrínsecos }\end{array}$ \\
\hline Glassman, (1992) & situaciones & \\
\hline Schein (2008) & $\begin{array}{l}\text { la teoría de } \\
\text { las anclas } \\
\text { de carrera }\end{array}$ & $\begin{array}{l}\text { Entendida como autopercepción que posee las personas basado en tres } \\
\text { elementos: habilidades, Actitudes y valores, Motivos o necesidades. }\end{array}$ \\
\hline (Escobar, 2019) & $\begin{array}{l}\text { Teoría de } \\
\text { adaptación } \\
\text { al trabajo }\end{array}$ & $\begin{array}{l}\text { Esta teoría permite la relación progresiva del empleado con el ambiente de } \\
\text { trabajo provocando una interacción adaptativa para el bienestar en común } \\
\text { (Escobar, 2019). }\end{array}$ \\
\hline
\end{tabular}

Fuente: Elaboración Propia (2020) 


\section{Journal of the Academy | 49|}

En este sentido, las teorías propuestas por varios autores sostienen que la satisfacción laboral genera libertad para discernir ¿Qué tan importante es la motivación de los colaboradores en las empresas?

En el estudio de la variable, prima el enfoque situacional, basado en el diseño del trabajo dentro del contexto laboral y en la importancia de las actitudes y proceder que ejecutan los trabajadores según (Pujol-Cols y Dabos, 2018).

Dentro de este orden de ideas y formulado el problema de investigación, se plantea como objetivo general del presente estudio: Determinar la relación que existe entre la gestión de talento humano y la satisfacción de los empleados de los bancos con mayor capital financiero de Guayaquil.

\section{MATERIALES Y MÉTODO}

El tipo de investigación fue cuantitativo, no experimental con corte transversal, descriptivo, correlacional; se aplicó una encuesta en línea a través de un cuestionario con 40 ítems con escala Likert, valorados con: (5) Totalmente de acuerdo, (4) De acuerdo, (3) Neutro, (2) Desacuerdo, (1) Totalmente desacuerdo; cuyos resultados fueron codificados en Excel y luego ingresados al programa estadístico SPSS.26 para el análisis de la correlación de las variables y dar respuesta a las hipótesis planteadas. El instrumento de recolección de información fue validado por expertos en el área.

La muestra fue de 370 trabajadores de las Instituciones bancarias con mayor capital financiero de la ciudad de Guayaquil, calculada con un margen de error 5\% y un nivel de confianza 95\%, implementando para el cálculo la fórmula estadística de población finita.

$$
\mathrm{n}=\frac{(1.96)^{2}(10000)(0.50)(0.50)}{(0.05) 2(10000-1)+(1.96)^{2}(0.50)(0.50)}=369,983704=370
$$

\section{Resultados}

Se pudo comprobar que la gestión de talento humano contribuye en la satisfacción laboral, según Frías (2020) el método de consistencia interna permitió estimar la fiabilidad de los 40 


\section{Journal of the Academy $|50|$}

ítems del instrumento de medida para la consistencia de datos el cual dio un valor significativo de 0.911 (Ver tabla 4) considerado un coeficiente de Alfa de Cronbach excelente (Mallery y George, 2003).

\section{Tabla 4}

Estadística de Fiabilidad

\begin{tabular}{ccrr}
\hline Alfa de Cronbach & $\begin{array}{c}\text { Alfa de Cronbach basada en elementos } \\
\text { estandarizados }\end{array}$ & $\begin{array}{c}\text { N de } \\
\text { elementos }\end{array}$ \\
\hline, 911 &, 912 & 40
\end{tabular}

Fuente: Resultados del programa SPSS. Elaboración Propia (2020)

Se realizó el análisis factorial exploratorio (AFE) de los datos obtenidos tomando en cuenta la aportación de López y Gutiérrez (2019) el muestreo de valoración del coeficiente de Kaiser, Meyer y Olkin cuya medida fue de 0.900 (Ver tabla 5), índice que permite determinar que es factible continuar con el análisis factorial, debido a que se pudo comprobar la correlación entre las variables mediante la prueba de esfericidad de Bartlett, con una estimación significativa del Chi-cuadrado de 0.000 demostrando un valor muy inferior al límite $<0.05$.

\section{Tabla 5}

\section{Prueba KMO y Bartlett}

\begin{tabular}{llr}
\hline Medida Kaiser-Meyer-Olkin de adecuación de muestreo &, 900 \\
\hline Prueba de esfericidad de Bartlett & Aprox. Chi-cuadrado & 4921,333 \\
\cline { 2 - 3 } & gl & 780 \\
& Sig. &, 000 \\
\hline
\end{tabular}

Fuente: Resultados del programa SPSS. Elaboración Propia (2020)

De acuerdo con los valores de la matriz de comunidades, anti-imagen y del análisis factorial de varianza total explicada se muestra como resultado 10 factores (Ver tabla 6) Utilizando el método de extracción con el análisis de componentes principales, cuyos resultados del programa SPSS de los componentes principales dieron un valor propio mayor que 1 de tal manera que permitió extraer los principales acordes a lo señalado. 
Journal of the Academy | $51 \mid$

\section{Tabla 6}

Varianza total explicada

\begin{tabular}{llll}
\hline \multirow{2}{*}{ Componente } & \multicolumn{2}{c}{ Autovalores iniciales } \\
\cline { 2 - 4 } & Total & \% de varianza & \% acumulado \\
\hline 1 & 10,094 & 25,235 & 25,235 \\
3 & 2,507 & 6,267 & 31,501 \\
4 & 1,711 & 4,277 & 35,778 \\
5 & 1,453 & 3,633 & 39,411 \\
6 & 1,291 & 3,229 & 42,640 \\
7 & 1,266 & 3,165 & 45,805 \\
8 & 1,222 & 3,056 & 48,861 \\
9 & 1,142 & 2,856 & 51,717 \\
10 & 1,070 & 2,674 & 54,392 \\
\hline
\end{tabular}

Fuente: Elaboración Propia (2020)

En base a los resultados de análisis de componentes principales en el método de extracción, se presenta el gráfico de sedimentación de los factores estudiados (Ver figura 1).

\section{Figura 1}

Gráfico de sedimentación de los componentes.

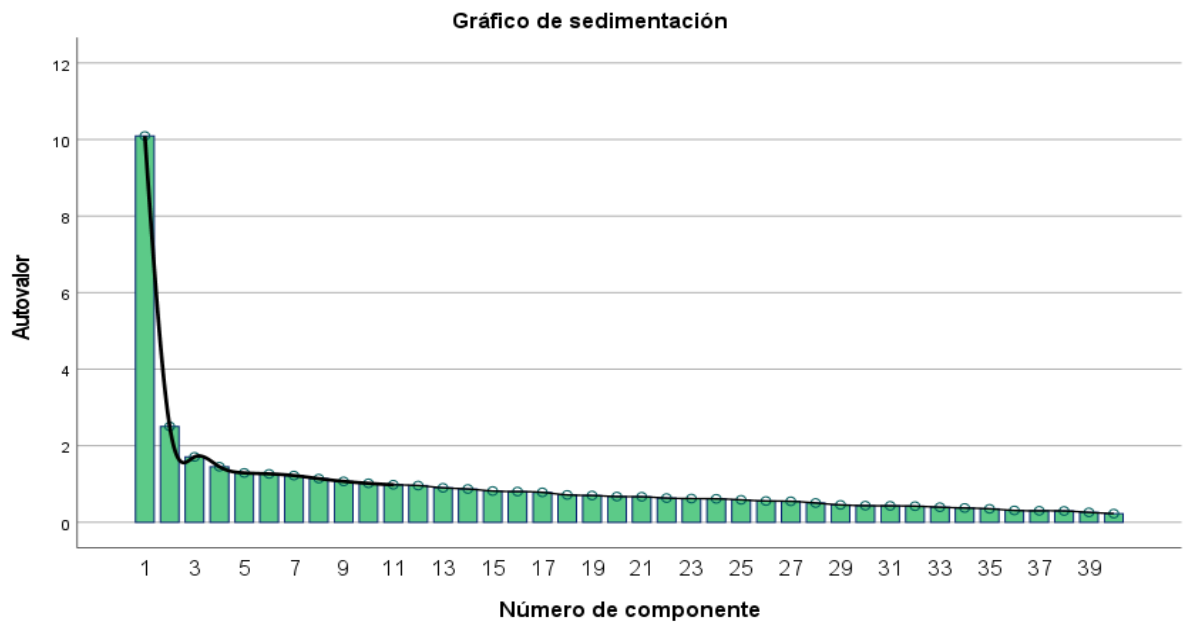

Fuente: Resultados del programa SPSS. Elaboración Propia (2020)

Teniendo en cuenta los resultados del programa SPSS en el estudio factorial explicada de la matriz de comunidades y matriz de componente rotado (Ver tabla 7) se puede observar la aplicación del método Varimax con normalidad de Kaiser para la extracción factorial con 


\section{Journal of the Academy | $52 \mid$}

máxima verosimilitud determinando que la rotación ha convergido en 10 iteraciones de los ítems garantizando la validación estadística de los componentes más importantes, en base al método de extracción y el análisis de componentes principales.

\section{Tabla 7}

Matriz de componentes rotado ${ }^{\mathrm{a}}$

\begin{tabular}{|c|c|c|c|c|c|c|c|c|c|c|}
\hline & 1 & 2 & 3 & 4 & 5 & 6 & 7 & 8 & 9 & 10 \\
\hline X1.1 & , 122 & ,256 & & & & & ,176 & ,626 & & \\
\hline X1.2 & & & & 190 & & 246 & ,105 & ,748 & & \\
\hline X1.3 & & & & & & & ,758 & ,214 & & ,108 \\
\hline $\mathrm{X} 1.4$ & & 693, & & & & & & & & \\
\hline X1.5 & & & & & & & & & & 871 \\
\hline X2.1 & 139, & ,341 & & 203 & & 584, & & & & \\
\hline $\mathrm{X} 2.2$ & & & & & & & & & 809 & \\
\hline $\mathrm{X} 2.3$ & 295 & 145, & 117, & , 180 & ,492 & & & & ,346 & \\
\hline $\mathrm{X} 2.4$ & ,140 & ,728 & & & ,155 & 146 & & & & \\
\hline X2.5 & ,372 & ,129 & & 262 & ,254 & ,431 & ,202 & , 125 & & \\
\hline X2.6 & , 169 & ,312 & 229 & & ,161 & 607, & & ,131 & & \\
\hline X3.1 & ,115 & ,749 & & 241 & ,171 & &,- 183 & ,109 & & \\
\hline X3.2 & ,300 & ,140 & ,420 & 437 & & ,102 & ,303 & & , 185 & \\
\hline X3.3 & ,284 & 503, & ,281 & 268 & & ,100 & ,159 & & ,200 & \\
\hline X3.4 & ,145 & ,727 & 187, & ,143 & , 182 & ,156 & & & & \\
\hline X3.5 & 296, & ,113 & 281 & ,141 & ,399 & ,272 & ,158 & 207 & & \\
\hline X4.1 & 229 & 379 & 267 & & & ,524 & & & & 146 \\
\hline $\mathrm{X} 4.2$ & ,321 & 690, & ,162 & & & ,118 & ,210 & & & \\
\hline $\mathrm{X} 4.3$ & & & 580, & ,212 & ,249 & ,348 & ,181 & & , 156 & \\
\hline X4.4 & & ,248 & & & ,315 & & ,146 & & & ,234 \\
\hline Y1.1 & ,156 & & 235 & & 638 & & & & & \\
\hline Y1.2 & ,339 & 193, & 245 & ,320 & ,254 & & & , 122 & ,309 & \\
\hline Y1.3 & ,262 & & 598 & & 283 & 189, & & & & \\
\hline Y1.4 & & ,147 & & ,765 & 139, & ,118 & & & & \\
\hline Y1.5 & ,159 & & ,743 & ,112 & & & & & & \\
\hline Y2.1 & 491 & ,314 & 219 & 186 & & & & ,210 & & \\
\hline Y2.2 & ,316 & ,115 & 235 & 433 & ,177 & & &, 173 & 298 & 257 \\
\hline Y2.3 & ,438 & & 209 & ,304 & ,247 & & & & & 163 \\
\hline
\end{tabular}


Journal of the Academy $|53|$

\begin{tabular}{|c|c|c|c|c|c|c|c|c|c|c|}
\hline $\mathrm{Y} 2.4$ & ,221 & ,151 & ,263 & ,376 & ,477 & & & & & \\
\hline Y2.5 & ,540 & 163 & 137, & ,515 & & & & & & \\
\hline Y2.6 & 489, & , 198 & ,418 & & & & , 126 & ,117 & & , 149 \\
\hline $\mathrm{Y} 2.7$ & 439, & ,222 & & ,449 & ,223 & & ,119 & & & \\
\hline Y3.1 & ,658 & & & & & , 169 & , 149 & & & \\
\hline Y3.2 & ,587 & ,137 & & & & & & & & \\
\hline Y3.3 & ,401 & & & , 148 & ,299 & , 195 & ,250 & & & \\
\hline Y3.4 & ,590 & & , 100 & 217, & & ,174 & & 123, & & \\
\hline Y3.5 & 609 & & ,139 & & , 120 & & , 135 & & & \\
\hline Y3.6 & ,572 & & & & 287 & ,267 & & & , 150 & \\
\hline Y3.7 & ,435 & ,174 & , 166 & & , 105 & & ,536 & & & \\
\hline Y3.8 & ,536 & & & ,207 & & ,327 & & & & , 144 \\
\hline
\end{tabular}

Fuente: Resultados del programa SPSS. Elaboración Propia (2020)

De igual manera se visualiza mediante el gráfico (Ver figura 2) la agrupación de los componentes en espacio rotado de las dimensiones de la variable independiente y dependiente verificando con base la estructura factorial para determinar la correlación que existen.

\section{Figura 2}

Gráfico de componentes en espacio rotado

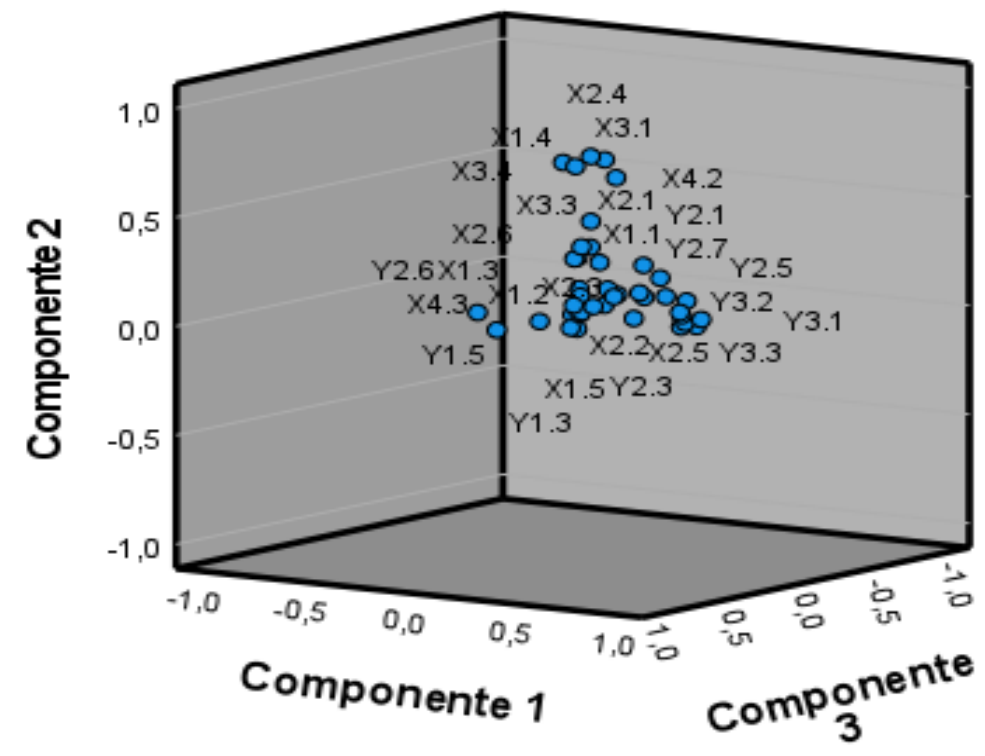

Fuente: Resultados del programa SPSS. Elaboración Propia (2020)

\section{Comprobación de hipótesis}

Con las frecuencias obtenidas en el estudio del Chi-Cuadrado se procedió a considerar el valor de probabilidad de los datos cuya frecuencia mínima esperada es $\mathrm{p}=<0.05$ para establecer si se 


\section{Journal of the Academy | $54 \mid$}

rechaza la hipótesis nula (H0) o se acepta la hipótesis alternativa (H1) de acuerdo con el valor significativo que se obtuvo en el análisis de datos.

Hipótesis general: La gestión de talento humano tiene relación directa con la satisfacción laboral de los empleados de los bancos con mayor capital financiero de Guayaquil. El nivel de significación obtenido es $\mathrm{p}=0.000$, siendo esta frecuencia $<0.05$ se procede a rechazar la hipótesis nula (H0) y por ende se admite la hipótesis alternativa (H1). En el análisis de las 1362 casillas $(99,8 \%)$ se obtuvo un valor menor que 5, lo que permite determinar que se alcanzó una asociación significativa de $\mathrm{p}=0,000$ (Ver tabla 8).

\section{Tabla 8}

Correlación entre la gestión de talento humano y satisfacción laboral

\begin{tabular}{llll}
\hline & Valor & gl & Significación asintótica (bilateral) \\
\hline Chi-cuadrado de Pearson & $3463,094^{\mathrm{a}}$ & 1292 &, 000 \\
\hline Razón de verosimilitud & 683,458 & 1292 & 1,000 \\
\hline Asociación lineal por lineal 165,314 & 1 &, 000 \\
\hline N de casos válidos & 370 & & \\
\hline
\end{tabular}

Fuente: Resultados del programa SPSS. Elaboración Propia (2020)

Hipótesis específicas: El nivel de significación obtenida en la correlación de las dimensiones de la variable gestión de talento humano y satisfacción laboral fue de p=0.000, considerando que se un valor $\mathrm{p}=<0.05$ se desecha la hipótesis nula $(\mathrm{H} 0)$ y se aceptan todas las hipótesis alternativas (H1), (Ver tabla 9).

\section{Tabla 9}

Correlación de las dimensiones de gestión de talento humano (GTH) con la satisfacción laboral mediante la Prueba de Chi-Cuadrado de Pearson

\begin{tabular}{lc|c|c|c}
\hline Hipótesis Alternativas & Valor & gl & $\begin{array}{c}\text { Signif. } \\
\text { Asint. } \\
\text { (bilateral) }\end{array}$ & Comprobación \\
\hline $\begin{array}{l}\text { H1 X1-Y Integración de GTH con satisfacción } \\
\text { laboral }\end{array}$ & $664,672^{\mathrm{a}}$ & 418 &, 000 & Se acepta \\
\hline $\begin{array}{l}\text { H2 X2-Y Organización de GTH con satisfacción } \\
\text { laboral }\end{array}$ & & &, 000 & Se acepta \\
\hline H3 X3-Y Formación de GTH con satisfacción laboral 1558,932 & 532 & 570 &, 000 & Se acepta \\
\hline H4 X4-Y Retención de GTH con satisfacción laboral 1155,471 & 456 &, 000 & Se acepta \\
\hline
\end{tabular}

Fuente: Resultados del programa SPSS. Elaboración Propia (2020) 


\section{Journal of the Academy | $55 \mid$}

A continuación, se muestra el Análisis Factorial Confirmatorio, los datos fueron procesados con el software estadístico SPSS-Amos 26.0 utilizando la gráfica de (path-diagram). Esta práctica está basada en Aráuz (2015) quien es considerado como precursor para los modelos que especifican relaciones estructurales entre las variables latentes.

En la figura 3 se muestran cargas factoriales mayores a 0.3 en valores absolutos de las estimaciones de las varianzas y correlaciones, considerando que $X / Y$ tuvo un valor de $p=0.95$ un coeficiente óptimo para establecer la conexión positiva y directa entre gestión de talento humano con satisfacción laboral de los colaboradores de los bancos con mayor capital financiero de Guayaquil.

\section{Figura 3}

Path Diagram. (AFC) Análisis Factorial Confirmatorio

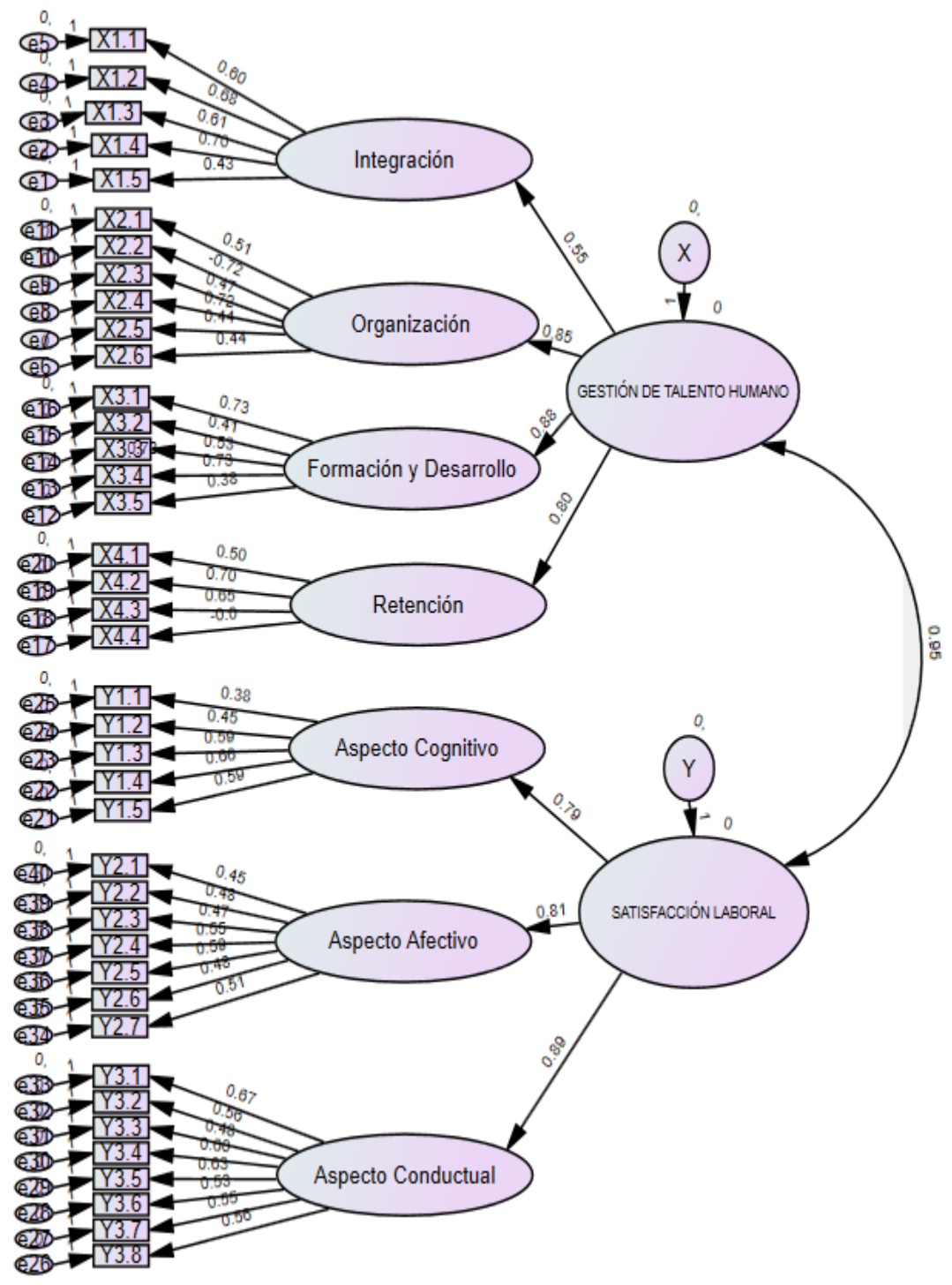

Fuente: Resultados del programa AMOS- SPSS. Elaboración Propia (2020) 


\section{Journal of the Academy $|56|$}

En la figura 4 se presenta un modelo factorial diferente para medir de manera estandarizada la métrica de los indicadores de factores latentes considerados para la prueba de hipótesis, el cual permitió visualizar las covarianzas y varianzas de las cargas factoriales consideradas como dimensiones de la variable $\mathrm{X}$, cuyos resultados fueron $\mathrm{X} 1 / \mathrm{Y}=0.79, \mathrm{X} 2 / \mathrm{Y}=0.60, \mathrm{X} 3 / \mathrm{Y}=0.70$, $\mathrm{X} 4 / \mathrm{Y}=0.55$, cuyas varianzas factoriales fueron significativos $(\mathrm{p}>0.3)$ en los valores obtenidos, de acuerdo a estas proyecciones se determina que: Las dimensiones de gestión de talento humano (X), integración (X1), organización (X2), formación y desarrollo (X3), retención (X4), inciden significativamente en la variable de satisfacción laboral (Y) de los trabajadores de los bancos con mayor capital financiero de Guayaquil.

\section{Figura 4}

Path Diagram. (AFC) Análisis Factorial Confirmatorio

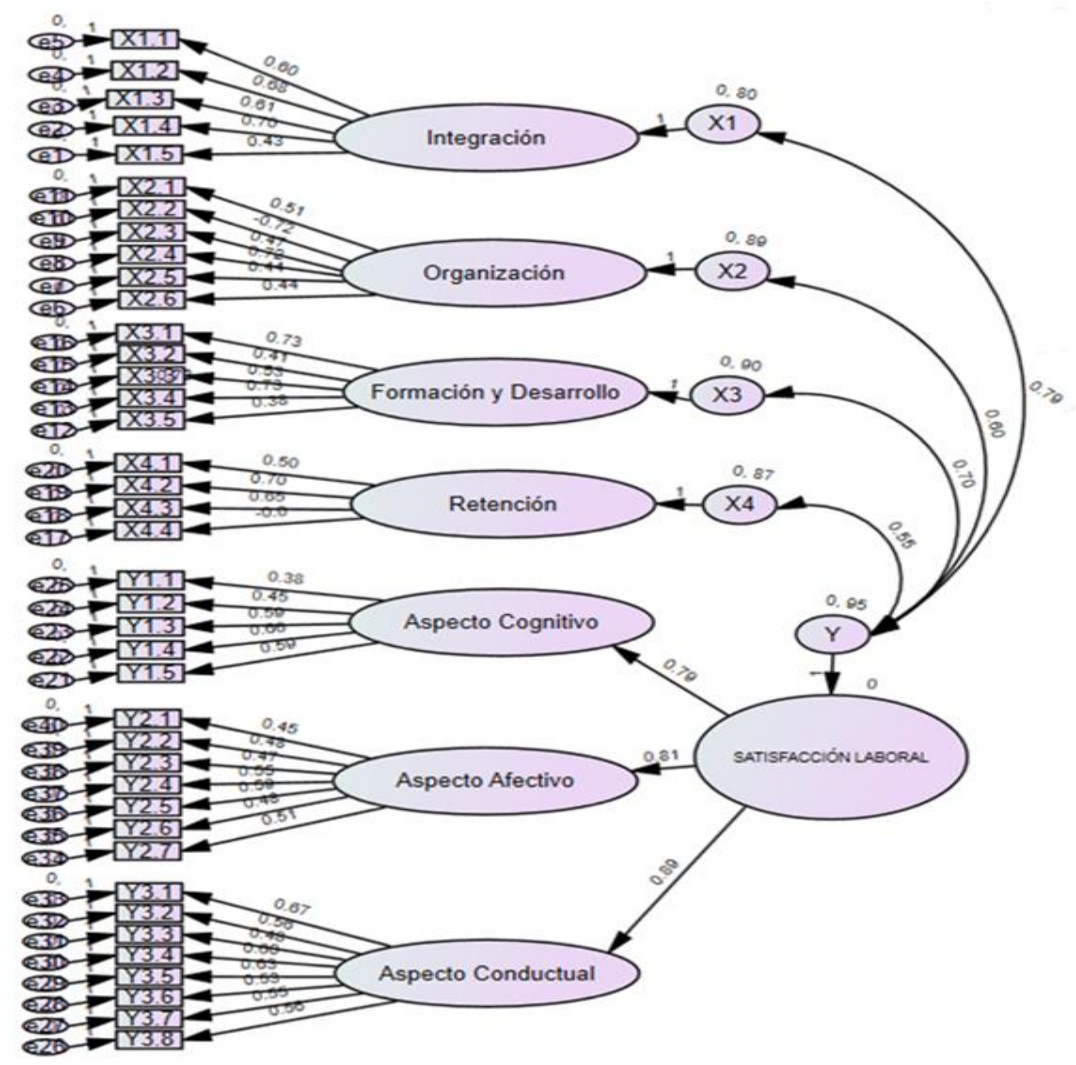

Fuente: Resultados del programa AMOS- SPSS. Elaboración Propia (2020)

\section{DISCUSIÓN DE RESULTADOS}

Se demuestra que la gestión de talento humano se relaciona positivamente con la satisfacción laboral de empleados de los bancos con mayor capital financiero de Guayaquil, con un 


\section{Journal of the Academy | $57 \mid$}

coeficiente de fiabilidad de Alfa-Cronbach de 0.911, cuyo muestreo de valoración del coeficiente de Kaiser, Meyer y Olkin fue de 0.900, en la prueba de esfericidad de Bartlett dio una estimación aproximada en el Chi-cuadrado un valor significativo $\mathrm{p}=<0.05$ de 0.000 , en el análisis factorial confirmatorio dio un valor absoluto de 0.95 en la estimación de la varianza de los factores, comprobando el desecho de la H0 y la estimación de la H1, coincidiendo con la investigación de (Daza, 2017; Ávila, 2018) quienes determinaron en su estudio una conexión significativa entre gestión de talento humano y satisfacción laboral del personal.

La integración se vincula de manera positiva con la satisfacción laboral de los trabajadores de los bancos con mayor capital financiero de Guayaquil, por medio de la prueba de esfericidad de Bartlett en el Chi Cuadrado de Pearson se obtuvo un valor estimado $\mathrm{p}=<0.05$ de 0.000 , en el análisis factorial confirmatorio se obtuvo un coeficiente significativo ( $p>0.3)$ de 0.79 estandarizado de varianza y covarianza de los factores latentes, determinando no aceptar la H0 y admitir positivamente la H1. Por su parte, Borrero (2019) considera que la integración constituye los procedimientos organizacionales idóneos para satisfacer las necesidades y requerimientos de la empresa.

La organización contribuye de manera positiva en la satisfacción laboral de los trabajadores de los bancos con mayor capital financiero de Guayaquil, demostrando por medio de la prueba de esfericidad de Bartlett en el Chi Cuadrado de Pearson un valor significativo $\mathrm{p}=<0.05$ de 0.000 , de forma semejante se obtuvo un coeficiente significativo ( $p>0.3$ ) de 0.60 estandarizado de varianza y covarianza de los factores latentes en el análisis factorial confirmatorio, determinando que se desestima la H0 y se reconoce a la H1, según Garbanzo (2015) la organización es considerada como un conjunto de coordinación de procesos específicos para alcanzar una meta determinada.

La formación y desarrollo se correlaciona de manera positiva con la satisfacción laboral de los trabajadores de los bancos con mayor capital financiero de Guayaquil, demostrando por medio de la prueba de esfericidad de Bartlett en el Chi Cuadrado de Pearson un valor significativo $p=<0.05$ de 0.000 , de forma semejante se obtuvo un coeficiente significativo $(p>0.3)$ de 0.70 estandarizado de varianza y covarianza de los factores latentes en el análisis factorial confirmatorio, estableciendo la refutación de la H0 aceptando la H1. También define a la capacitación como sinónimo de formación, desarrollo y aprendizaje del personal de una organización para complementar los conocimientos y desempeñar su labor (Díaz et al., 2020). 


\section{Journal of the Academy $|58|$}

La retención se conecta de manera positiva con la satisfacción laboral de los trabajadores de los bancos con mayor capital financiero de Guayaquil, demostrando por medio de la prueba de esfericidad de Bartlett en el Chi Cuadrado de Pearson un valor significativo $\mathrm{p}=<0.05$ de 0.000 , de forma semejante se obtuvo un coeficiente significativo ( $p>0.3$ ) de 0.55 estandarizado de varianza y covarianza de los factores latentes en el análisis factorial confirmatorio, procediendo a rechazar la H0 y aceptar la H1. Para Prieto (2013) la retención de personal es importante para integrar no tan solo una organización sino también un conjunto de procedimientos que permiten alcanzar la productividad de la organización.

\section{CONCLUSIONES}

Se pudo comprobar a través del presente estudio investigativo que las variables y dimensiones de gestión de talento humano incide notablemente en la variable y dimensiones de la satisfacción laboral mediante un análisis respectivo que se realizó con los datos absolutos obtenidos de los usuarios entrevistados, los cuales fueron representados a través de valores significativos y aceptables del coeficiente alfa cronbach (0.911), de pruebas KMO y Barllett $(, 000)$ conjuntamente con el análisis factorial confirmatorio y exploratorio de componentes principales bajo el método de Varimax - Kaiser cuya rotación convergido fue10 iteraciones importantes.

Teniendo en cuenta la incidencia de la gestión de talento humano y satisfacción laboral, se puede concluir que la gestión de talento humano cumple un rol interesante en toda empresa para la toma de decisiones dando soluciones factibles, eficaces y eficientes que promuevan satisfacción laboral en la empresa. Ante las proyecciones de los resultados podemos determinar que se logró cumplir con el objetivo general de esta investigación determinando así la relación directa y positiva entre la gestión de talento humano y satisfacción laboral de los empleados de los bancos con mayor capital financiero de Guayaquil.

En consecuencia, toda organización debe contar con gestión de talento humano compuesta por diferentes métodos y sistemas prácticos para brindar satisfacción y una cultura organizacional eficiente y pacífica capaz de enfrentar retos de modernización al momento de recrear una excelente gestión empresarial. 


\section{Journal of the Academy | $59 \mid$}

Por lo tanto, este estudio investigativo servirá para dar continuidad al análisis de los diferentes tipos de dimensiones en la gestión de talento humano y satisfacción laboral, desde las teorías aplicadas, encaminando hacia diversas líneas de investigación, sirviendo como orientación no tan solo a las instituciones financieras, sino también a otras instituciones públicas y privadas a nivel local, nacional e internacional, lo cual permitirá que el análisis de las variables, sean relacionadas con otras fuentes de estudios.

\section{REFERENCIAS BIBLIOGRÁFICAS}

Aráuz, A. (2015). Aplicación del análisis factorial confirmatorio a un modelo de medición del rendimiento académico en lectura. Revista de Ciencias Económicas, 33(2), 1-28. http://dx.doi.org/10.15517/rce.v33i2.22216

Ávila, J. (2018). Gestión del talento humano y satisfacción laboral del personal asistencial de salud, Hospital de Barranca-Cajatambo, 2018. [Tesis de Maestría, Universidad Nacional José Faustino Sánchez Carrión]. http://repositorio.unjfsc.edu.pe/bitstream/handle/UNJFSC/2389/AVILA\%20DEL\%20VALLE \%20JOSE.pdf?sequence=1\&isAllowed $=\mathrm{y}$

Barros, M., Barros, B., Moy-Sang, S. y Romero, W. (2017). La calidad de gestión de procesos cognitivos y el desarrollo educativo. [I Congreso de Ciencia, Sociedad e Investigación Universitaria, Pontífica Universidad Católica del Ecuador]. https://repositorio.pucesa.edu.ec/bitstream/123456789/2084/2/La\%20Calidad\%20de\%20Gesti \%C3\%B3n\%20de\%20Procesos.pdf

Borrero, R. (2019). Procesos de gestión del talento humano en el sector educativo gerencial de Colombia. Revista Arbitrada Interdisciplinaria - Fundación Koinonía, 4(7), 1-10. http://portal.amelica.org/ameli/jatsRepo/105/105590017/html/index.html

Chacón, L. (2016). Diseño e implementación de un modelo de gestión de talento humano basado en competencias, para la empresa Crédi útil de la ciudad de Latacunga. [Tesis de Magister, Escuela Politécnica Nacional]. https://bibdigital.epn.edu.ec/bitstream/15000/16117/1/CD-7118.pdf

Chiang, M. y Ojeda, J. (2013). Estudio de la relación entre satisfacción laboral y el desempeño de los trabajadores de las ferias libres. Contaduría y Administración, 58(2), 39-60. https://doi.org/10.1016/S0186-1042(13)71209-9

Cruz, J. (2018). La calidad de vida laboral y el estudio del recurso humano: una reflexión sobre su relación con las variables organizacionales. Revista Pensamiento y Gestión, 45(1), 1 20. https://www.redalyc.org/jatsRepo/646/64659525004/html/index.html

Cruz, P., Rojas, S., Vega, G. y Villegas, Y. (26 de julio de 2002). Capital humano y gestión por competencias. Gestiopolis. https://www.gestiopolis.com/capital-humano-y-gestion-porcompetencias/ 


\section{Journal of the Academy $|60|$}

Daza, A. (2017). Gestión del talento humano y satisfacción laboral del personal de enfermería de neonatología del Hospital Arzobispo Loayza 2015. [Tesis de maestría, Universidad César Vallejo. Perú]. http://repositorio.ucv.edu.pe/handle/20.500.12692/21810

Díaz, J., Ledesma, M., Rojas, S. y Díaz, L. (2020) Los cuatro saberes de la educación como formación continua en las empresas. Fides et Ratio - Revista de Difusión cultural y científica de la Universidad La Salle en Bolivia 19(19). http://www.scielo.org.bo/scielo.php?script=sci_arttext\&pid=S2071-081X2020000100003

El Universo. (23 de diciembre de 2020). Los diez bancos de Ecuador con más utilidades en el 2020. Diario El Universo, Economía. https://www.eluniverso.com/noticias/2020/12/23/nota/8711788/diez-bancos-ecuador-masutilidades-2020/

Montoya, C y Boyero, M. (2016). El recurso humano como elemento fundamental para la gestión de calidad y la competitividad organizacional. Revista Científica "Visión de Futuro", 20(2), 1-20. https://www.redalyc.org/jatsRepo/3579/357947335001/html/index.html

Escobar, M. (2019). Gestión del talento humano y la satisfacción laboral del Personal Administrativo de la Universidad Nacional de Educación Enrique Guzmán y Valle, distrito de Lurigancho-Chosica, 2017. [Tesis de maestría. Universidad Nacional de Educación, Lima]. http://repositorio.une.edu.pe/bitstream/handle/UNE/2651/TM\%20ADGp\%204238\%20E1\%20-\%20Escobar\%20Guevara.pdf?sequence=1\&isAllowed=y

Frías, D. (2020). Análisis de la consistencia interna de las puntuaciones de un instrumento de medida. [Tesis de Doctorado, Universidad de Valencia. España]. https://www.uv.es/ friasnav/AlfaCronbach.pdf

Garbanzo, G. (2015). Desarrollo organizacional y los procesos de cambio en las instituciones educativas, un reto de la gestión de la educación. Revista Educación, 40 (1), 67-87, https://www.redalyc.org/jatsRepo/440/44043204005/html/index.html

Grijalva, A., Palacios, J., Patiño, C y Tamayo, D. (2017). Los factores asociados a la satisfacción laboral en Ecuador en 2007 y 2015 utilizando la Encuesta Nacional de Empleo, Desempleo y Subempleo. Revista de Análisis Estadístico, 13(1), 1-45. https://www.ecuadorencifras.gob.ec/documentos/webinec/Revistas/Analitika/Anexos_pdf/Analit 13/1.pdf

López, M. y Gutiérrez, L. (2019). Cómo realizar e interpretar un análisis factorial exploratorio utilizando SPSS. Revista d'Innovació $i$ Recerca en Educació, 12(2), 1-14. http://doi.org/10.1344/reire2019.12.227057

Mallery, P. y George, D. (2003). SPSS for Windows step by step: A simple guide and reference. 11.0 Update (4th ed). Ally \& Bacon.

Manene, L. (16 de septiembre de 2012). Motivación y satisfacción en el trabajo y sus teorías. Biblioteca de Conocimientos, Gestión Del Personal, Mejora Continua. http://www.luismiguelmanene.com/2012/09/16/la-motivacion-y-satisfaccion-en-el-trabajo-y$\underline{\text { sus-teorias/ }}$ 


\section{Journal of the Academy | $61 \mid$}

Manjarrez, N., Sablón, N. y Ortega, V. (2016). La evolución de la teoría sobre la gestión de los recursos humanos. Gestión de los recursos humanos: Un abordaje teórico. Revista Eumednet 1(1), 1-19. http://www.eumed.net/cursecon/ecolat/ec/2016/rrhh.html

Martínez, K. (2011). Los contenidos procedimentales en el desarrollo del pensamiento crítico de los estudiantes del tercer ciclo de derecho penal de la Facultad de Jurisprudencia de la Universidad Católica, período 2009- 2010. [Tesis de maestría, Universidad Técnica de Ambato]. https://repositorio.uta.edu.ec/bitstream/123456789/2487/1/t_ma_dyc_812.pdf

Mejía, M y Tómala, M. (2017). Estrategias didácticas con el uso de la tecnología para mejorar el nivel de aprendizaje cognitivo en los estudiantes. [Tesis de maestría, Universidad Laica]. https://repositorio.uleam.edu.ec/bitstream/123456789/883/1/ULEAM-CCAD-0009.pdf

Meneses, M y Bendezú, H. (2017). La afectividad y el aprendizaje de los estudiantes del primer grado de secundaria de la Institución Educativa $N^{\circ} 23008$ "Ezequiel Sánchez Guerrero" de Ica. [Tesis de Ingeniero Agrónomo, Universidad Nacional de Huancavelica, Perú]. http://repositorio.unh.edu.pe/handle/UNH/1285

Mondragón, A., García, E. y Chávez, E. (2017). Modelos de gestión de recursos humanos. Revista Científica TEPEXI de la Escuela Superior Tepeji del Río, Universidad Autónoma del Estado de Hidalgo 8(4), 1-100. https://www.uaeh.edu.mx/scige/boletin/tepeji/n8/a13.html

Pérez, O. (23 de mayo de 2016). Gestión del talento humano en la empresa y su importancia. Blog PeopleNext. https://blog.peoplenext.com.mx/gestion-del-talento-humano-en-la-empresay-su-importancia

Pilatasig, C. (2020). Análisis de la satisfacción laboral de los docentes del Instituto De Educación Especial Juan Pablo II, en la ciudad de Esmeraldas, año lectivo 2019 - 2020. [Tesis de grado, Pontífica Universidad Católica del Ecuador] https://repositorio.pucese.edu.ec/handle/123456789/2068\#: :text=La\%20satisfacci\%C3\%B3n \%201aboral\%20se\%20entiende, los\%20diferentes\%20aspectos\%20del\%20mismo

Prieto, P. (2013). Gestión de talento humano como estrategia para retención del personal. [Tesis de especialista, Universidad de Medellín]. https://repository.udem.edu.co/handle/11407/160

Pujol-Cols, L. y Dabos, G. (2018). Satisfacción laboral: una revisión de la literatura acerca de sus principales determinantes. Estudios Gerenciales, 34(146), 3-18. https://doi.org/10.18046/j.estger.2018.146.2809

Ramos, R. (2018). Gestión del talento humano y desempeño laboral en la Pastelería Miraflores S.A.C. [Tesis de Maestría, Universidad César Vallejo]. http://repositorio.ucv.edu.pe/handle/20.500.12692/13847

Superintendencia de Bancos del Ecuador. (junio de 2018). Instituciones financieras con mayores utilidades. Superintendencia de Bancos del Ecuador. http://estadisticas.superbancos.gob.ec/portalestadistico/portalestudios/wpcontent/uploads/sites/4/downloads/2019/03/reporte_estabilidad_jun_2018.pdf 